\title{
LES SITES MÉMORIELS ET LEURS ESPACES. ÉLÉMENTS POUR UNE ANALYSE IN SITU
}

\author{
OS SÍTIOS DE MEMÓRIA E SEUS ESPAÇOS. ELEMENTOS PARA UMA ANÁLISE IN SITU
}

\author{
Philippe Mesnard ${ }^{1}$
}

RÉSUMÉ: De nos jours, les musées liés à des processus traumatiques ou qui ont marqué l'histoire de l'humanité, sont devenus des façons différentes de vivre l'expérience de la visite. Par leur scénographie, ils induisent un comportement participatif, au lieu d'une attitude passive et sans engagements par le visiteur. C'est dans le fonctionnement de ces lieux institutionnels, tant sous la dimension interne qu'en relation avec les autres espaces muséaux, que cet article cherche à présenter quelques hypothèses d'interprétation.

Mots clés: musées de mémoire, scénographie, homogénéisation, normalisation, hétérotopie.

RESUMO: Na atualidade, os museus relacionados a processos traumáticos ou que marcaram a história da humanidade, transformaram-se em formas diferenciadas de viver a experiência da visita. Através de suas cenografias, induzem a um comportamento participativo, ao invés de uma atitude passiva e descomprometida. É na maneira como esses lugares institucionais funcionam, tanto na perspectiva interna, como nas relações com outros espaços museais, que o presente artigo busca apresentar algumas hipóteses de interpretação.

Palavras-chave: museus de memória, expografia, homogeneização, normatização, heterotopia.

En parcourant des lieux dédiés à la mémoire d'événements ou de processus ayant marqué l'histoire de l'humanité, d'une nation ou d'une communauté, les visiteurs évoluent aujourd'hui dans des scénographies qui les sollicitent pour être plus que des touristes ou de simples consommateurs culturels. Ils ne sont pas non plus considérés comme des êtres passifs à qui l'institution mémorielle livre un message, mais comme des acteurs s'impliquant par le biais de dispositifs qui suscitent en eux le sentiment d'être concernés et, parfois même, d'être de véritables sujets au milieu des représentations qu'ils traversent. Une fois sortis de ces sites, ils recouvrent une certaine autonomie qui leur laisse la liberté de traverser des espaces pourtant de plus en plus balisés par des mémoires tantôt hétéroclites et disparates, tantôt homogènes. C'est à la façon dont ces lieux institutionnels fonctionnent à l'intérieur aussi bien qu'entre eux, dans l'espace urbain et en rapport à ceux qui les arpentent, que la présente étude s'intéresse et tente de poser des hypothèses d'interprétation.

\footnotetext{
${ }^{1}$ Docteur en Littérature Comparée. Professeur de littérature comparée à l'Université Clermont Auvergne/Institut Universitaire de France.
} 


\section{Focalisation interne}

Visiter ces espaces mémoriels nous engage dans un double processus d'acquisition d'une connaissance, d'une part, et d'expériences émotionnelles, d'autre part, que favorisent et parfois conditionnent les scénographies suivant lesquelles ces espaces et ce qu'ils représentent sont ordonnés. Même dans les configurations les plus radicales, ce partage entre connaissance et émotion n'est pas strictement binaire, il y a toujours, soit une documentation minimum procurant des repères objectifs sur le sujet, soit une dimension affective, même si elle est réduite au peu qu'expriment certaines pièces placées dans un cadre intentionnellement neutre. Dans tous les cas, le jeu du cognitif et de l'affectif participe à et d'un dosage que l'on peut appeler une économie des représentations mémorielles.

Le propre des espaces qui privilégient le cognitif est la clarté et le volume. Ainsi, les trois musées de Malines, situé en Flandres occidentales belges, de Péronne en France et de La Junquera en Catalogne espagnole sont de remarquables exemples de ce parti pris, d'autant que chacun a un objet très différent. Le premier, la Kazerne Dossin, traite du génocide des Juifs et de la persécution de ceux-ci en Belgique avec un prolongement sur la question des droits de l'homme. Le second, L'Historial de la Grande Guerre, au cœur de la Somme, met l'accent sur les combattants et la violence de 1914-1918. Le dernier, le Museu Memorial de l'Exili (MUME), concerne principalement le déplacement forcé, dénommé Retirada, des populations espagnoles et la retraite des combattants devant l'avancée des troupes franquistes à la fin de la guerre d'Espagne. Leurs différences thématiques rendent encore plus frappante la similitude de ces mises en scène qui insistent toutes, avec une orientation pédagogique poussée, sur l'information, l'explication et la nécessité de faire comprendre à travers du texte (notices, panneaux explicatifs, sources consultables sur écran) et du discours oral (témoignages enregistrés, audioguide) de quelle violence il s'est agi, tout en mettant les visiteurs à distance des risques de se faire prendre par l'émotion.

En revanche, d'autres institutions se caractérisent par la dimension affective de leur scénographie. L'espace intérieur est alors si sombre et si étroit qu'il semble être le négatif photographique des précédents. Le mémorial Yad Layeled du kibboutz Lohamei Hagetaot au nord d'Israël, le musée In Flanders Field de Ypres en Belgique et le mémorial de Vassieux en Vercors illustrent, chacun à sa façon, cette configuration dans laquelle le visiteur se retrouve exposé à un environnement sonore et visuel immersif le privant de cette distance que réclame l'intellect pour comprendre ce qu'on lui montre. Les assassinats de Juifs et plus particulièrement des enfants pour le premier; le martyr de la ville d'Ypres et des soldats britanniques soumis aux gaz de l'armée allemande en 1917 pour le second, et le massacre des habitants du village de Vassieux lors de l'attaque des parachutistes allemands contre le maquis du Vercors en 1944 sont des crimes dont la cruauté dépasse ce que l'on entend communément par violence de guerre. La dimension émotionnelle domine le visiteur pour non seulement lui présenter ce qu'il voit, mais aussi lui faire ressentir l'effroi dès lors qu'il circule en suivant le parcours toujours très balisé de la scénographie. Sur ce plan, un des dispositifs du nouveau musée de la Tour de l'Yser, à Dixmude n'a probablement d'égal, réalisant la volonté de produire un effet-choc sur les visiteurs, que celui dédié à l'attentat de la ville d'Oklahoma City qui a eu lieu le 19 avril 1995. Un des étages de la Tour de l'Yser plonge les visiteurs - ayant préalablement été prévenus que ceux qui seraient sujets à une crise d'épilepsie doivent se rendre à l'étape suivante - dans le noir complet avec pour seul guide de petites lumières au sol. Plus ils avancent, plus ils entendent un souffle haletant qui aurait pu être celui d'un soldat britannique 
succombant à une attaque au gaz. Au musée d'Oklahoma City, les visiteurs sont placés dans une salle avec une simulation d'explosion et des projections des visages des victimes sur les murs (STURKEN, 2011 e 2007). Dans chacun de ces cas, dispositifs scénographiques et parcours s'imposent comme des mots clés non seulement de la muséographie, mais surtout comme des enjeux de la représentation mémorielle en situation spatiale. Ainsi, dans les versions les plus affectives de celle-ci, la liberté de choix du visiteur est limitée par le trajet qu'il doit emprunter pour avancer sans guère d'alternative, sauf à s'extraire intentionnellement au dehors d'un dispositif qui lui impose son autorité. Plus l'émotion est déterminante, plus le parcours est étroit et directif.

L'on éprouve aussi cette impression dans un autre lieu que je n'ai pas encore mentionné, l'United States Holocaust Memorial Museum (USHMM) de Washington. Même si cette institution ne néglige évidemment pas l'information historique du génocide des Juifs par les nazis et leurs collaborateurs, la scénographie de l'exposition permanente n'en combine pas moins, pour le visiteur, l'expérience inquiétante d'une montée par un ascenseur (seule voie d'accès aux salles) et un jeu d'ombre et de pâle clarté aussi complexe que le dédale de ses couloirs, l'un d'entre eux étant caractérisé par l'odeur de moisissure d'un amas de chaussures de déportés. En effet, le rapport émotionnel d'une exposition passe par l'image et par cette mimésis toute particulière de l'identification individuelle à ceux qui, volontairement ou non, ont été engagés dans l'histoire traitée à l'intérieur de l'espace de représentation (MESNARD, 2012). En ce sens, le musée de l'Holocauste de Washington, ouvert en 1993, a innové en invitant chaque visiteur à prendre la carte d'identité d'une victime juive pour, à travers les salles d'exposition, en suivre la destinée la plupart du temps fatale. Nul besoin d'insister sur la charge affective que peut revêtir une telle expérience identificatoire.

Des procédés similaires se retrouvent dans différents musées et expositions itinérantes et continuent à être amplement exploités, comme au musée In Flanders Field d'Ypres où tout incite à l'empathie avec les victimes et à un confus rapprochement des identités. Il est néanmoins intéressant de remarquer que, pour radicalement opposées que soient la mise en scène de ce dernier musée et celle de la Kazerne Dossin, les deux utilisent abondamment des consoles multimédia similaires pour trouver l'identité de victimes et retracer leur parcours. Moins ludique effectivement que dans In Flanders Field où chaque visiteur se voit remettre un bracelet permettant, après enregistrement, d'être reconnu dès qu'il le pose sur un lecteur, les moniteurs de Dossin n'en sont pas moins troublants. Ils font apparaître tout un jeu de vignettes et d'informations sur les victimes dans un style rétro en contraste complet avec l'architecture.

L'argument que la technologie soit neutre, valant pour toute scénographique aussi bien à dominante documentaire qu'émotionnelle, n'est ici ni suffisant, ni satisfaisant. Il s'agit de tisser un lien avec le spectateur en l'introduisant, par un acte pratique nécessitant une implication de soi, dans un processus d'appropriation empathique et/ou intellectuel. Le corps et la capacité à agir sont fortement convoqués et incités. C'est ce que l'on pourrait appeler, s'il s'agissait du langage de la communication médiatique ou du marketing, la finalité conative de ces dispositifs. Participer au jeu et faire des choix renforcent la relation au processus dans lequel on est entré et constitue une véritable expérience. Mettant en pratique, non pas à l'échelle de la langue mais de l'espace, les catégories rhétoriques du docere et du movere (la documentation et l'émotion), la muséographie mémorielle répond aux exigences des variations d'une économie représentationnelle où l'expérience vécue par le visiteur et la personnification du parcours se sont imposées comme point de médiation conative entre le cognitif et l'affectif. Tantôt le parcours est dominé par l'interprétatif, tantôt il émeut le visiteur qui finit par devenir un véritable acteur de l'exposition (parfois même son véritable acteur).

Dans les paramètres de cette économie et pour en rendre compte plus précisément, il 
faut aussi considérer comment sont utilisés les objets qui fonctionnent comme des catalyseurs majeurs des émotions, des identifications et de jeux de rôle élémentaire auxquels se prêtent les visiteurs (STURKEN, 2007). Certains espaces sont placés sous leur règne : le petit chausson de nourrisson de Yad Layeled et les vêtements de jeunes enfants juifs dans une des vitrines du musée juif de Berlin versus la mitraillette Sten du Musée de l'insurrection à Varsovie que tant se sont surpris à épauler. James Young estimait que le véritable monument à la destruction des Juifs d'Europe de Berlin tenait bien plus aux débats qui en ont animé la réalisation pendant une dizaine d'années, qu'au «produit fini» que leur rigidité finit par absorber. En transposant ce principe, ne pourrait-on pas concevoir au-delà des murs et de la rigidité des monuments ${ }^{2}$, les espaces mémoriels comme des sites eux-mêmes en mouvement?

Guidé et maîtrisé, ou non, le parcours du visiteur a jusque-là été pensé au-dedans de zones spécifiquement définies par leur identité fonctionnelle et référentielle, comme si la mémoire n'était qu'intérieure, et qu'elle ne procédait que par rétention (d'ailleurs, pour qualifier une des caractéristiques de la conception hégémonique de la mémoire, ne pourrait-on pas proposer le néologisme de mémoire rétentive?). Que dire alors des trajets qui mènent à ces endroits en nous faisant croiser d'autres lieux, parfois représentatifs d'une même mémoire, mais aussi parfois représentatifs d'autres mémoires? Quelles relations entretiennent ces différentes mémoires entre elles? Alors que depuis les années 2000, les institutions mémorielles se sont multipliées dans l'espace public que l'on habite - au niveau de mémoires historique, sociale et patrimoniale -, ne néglige-t-on pas les relations urbaines, voire périurbaines qui les lient les unes aux autres?

\section{Vue du dehors}

Étendre la notion d'espace mémoriel permet de donner un nouvel éclairage à ce phénomène de plus en plus présent dans notre culture et, indissociablement avec lui, aux flux qu'il draine. Pas plus que l'hôpital ou la prison pour l'administration de la société (FOUCAULT, 1972 e 1975) et de ses marges, ni l'église de façon encore flagrante dans la topographie du village, le musée ni le mémorial ne règnent en solitaire; ils appartiennent au maillage social, culturel et politique qui, à l'instar de la présence physique du visiteur, engage le corps de la société et dont l'on fait l'expérience en tant que bien commun. «Nous ne vivons pas à l'intérieur d'un vide qui se colorerait de différents chatoiements, nous vivons à l'intérieur d'un ensemble de relations qui définissent des emplacements irréductibles les uns aux autres et absolument superposables» (FOUCAULT, 1984). Appréhender cette dimension de la mémoire, laquelle est pleinement à entendre comme une restitution topographique du passé collectif dans le présent du vivre ensemble, demande donc méthodologiquement de se défocaliser de l'intérieur des sites pour porter l'attention sur la cartographie que dessinent les entités mémorielles (musées, mémoriaux, monuments, installations d'artistes et commémoratives...) en dialogue ou en tension les unes avec les autres.

Je me limite ici à poser quelques hypothèses sous-tendues par des exemples pour maintenir l'intention prospective de ce texte. Visite-t-on les lieux mémoriels institués sans

\footnotetext{
${ }^{2}$ Que l'on ne voit pas ici une position iconoclaste qui serait anti- ou contre-monumentale, mais plutôt une critique de l'immobilité monumentale qui, même si l'on est passé, en Occident et plus particulièrement en Europe, du surfiguratif héroïsant à l'anonymat des victimes vulnérables, n'a guère évolué, malgré les avertissements de quelques artistes des années 1980-1990 dont n'ont été gardés que les artifices les plus facilement reproductibles et consommables. Voir Philippe Mesnard, «Comment rendre visible l'effacement des traces», in Ewa Berard et Luba Jurgenson, Une histoire sans traces? Le patrimoine matériel russe et la culture mémorielle en Europe, Paris, Petra, 2016.
} 
enregistrer ou percevoir, ne serait-ce qu'indirectement ou de façon subliminale, le tissu spatial dans lequel ils sont implantés et dont ils composent eux-mêmes un des motifs ? S'épanouissentils chacun individuellement comme des singularités, ou bien n'y a-t-il pas un plan, un espace, voire un monde possible qui les accueille et se co-construit avec eux, ouvrant comme à une quatrième dimension de la mémoire faite d'interconnexions?

En préalable, il faut prendre en compte que parmi ces interconnexions, le visiteur assure une fonction de révélateur mettant en évidence la marge ou l'absence de marge qui lui est laissée pour qu'il prenne ou non des initiatives, qu'il élabore ou non un jugement autoréflexif. Se pose ainsi la question du rapport entre, d'un côté, les stratégies des entrepreneurs mémoriels au niveau de l'espace urbain avec des stratégies qui relèvent aussi bien des politiques mémorielles que du marketing ${ }^{3}$ et, de l'autre, les tactiques que les visiteurs ont la ressource de s'inventer pour circuler sans emprunter nécessairement les voies toutes tracées pour eux, réagissant aux précédentes stratégies par des comportements et des défenses ${ }^{4}$ tactiques que Michel de Certeau (1990) assimile à du braconnage, ce qui permet de préserver une variété de motivations entre le «strict» pèlerinage et la "pure» consommation touristique - sachant que celle-ci ne peut se limiter à cette sorte de curiosité hédoniste et parfois malsaine à laquelle on réduit souvent le Dark tourism ${ }^{5}$. D'une certaine manière, on pourrait comparer ceux qui déambulent au XXI ${ }^{\mathrm{e}}$ siècle ces espaces aux flâneurs baudelairiens tels qu'ils ont été revisités par Benjamin $^{6}$, la culture ayant su ouvrir de grands boulevards de la mémoire dans l'espace public qui prolongent notre imagination (celle-ci comptant elle-même ses propres développements et prothèses virtuels sur le web). Toutes ces relations dont il faut maintenir l'hétérogénéité pour en préserver ou en augmenter la richesse, s'animent d'échanges et de commerces dans le jeu desquels la mémoire recouvre sa véritable et initiale fonction de médium ${ }^{7}$ - pour reprendre également une notion benjaminienne - , de passeuse pour ces nouveaux flâneurs en quête des éclats sombres de ce qui a eu lieu.

Tâchons maintenant de préciser en quoi ces aspects se différencient les uns des autres. Le tissu plus ou moins serré de cette topographie s'avère être comme un feuilletage de plusieurs niveaux d'agencement par lesquels les sites trouvent une place et, parfois, dans lesquels ils se révèlent pris et enserrés. On peut ainsi être étonné par l'homogénéité et la stéréotypie qui uniformisent certains, reflétant combien leurs concepteurs ou bien manquent d'imagination, ou bien sont contraints par le cahier des charges qui leur est imposé, ou bien encore ne prennent aucun risque face à l'objet incertain qui les motive. En revanche, il est une autre sorte d'unité qui s'opère non pas par la standardisation, mais par l'adhésion à un discours institutionnel provenant tantôt des représentants des pouvoirs publics, tantôt de groupes privés

\footnotetext{
${ }^{3}$ Pour une étude de terrain sur l'influence de la muséification menée par des entrepreneurs mémoriels publics et privés sur la Catalogne pyrénéenne, voir Camila del Marmol, "Through Other Times: The Politics of Heritage and the Past in the Catalan Pyrenees", in David Picar et Michael A. Di Giovine (dir.), Tourism and the Power of the Otherness. Seductions of Difference, Bristol, Channel View, 2014, pp. 31-52.

${ }^{4}$ Ces défenses peuvent être néanmoins très réactives et traditionnalistes, quand les politiques publiques sont ouvertes et progressistes.

${ }^{5}$ Pour une approche plus fine que la critique, généralement francophone, de ces phénomènes: John Lennon et Malcolm Foley, Dark Tourism. The attraction of death and disaster, Londres, Thomson, 2000; Richard Sharpley et Philip R. Stone, The Darker Side of the Travel. The Theory and Practice of Dark Tourism, Bristol, Channel View Publications, 2009. Annette Becker et Charles Forsdick, dossier: «Tourisme mémoriel : la face sombre de la terre?», Mémoires en jeu, Paris, n 3, mai 2017, pp. 43-102.

${ }^{6}$ Walter Benjamin, «Le Flâneur», Charles Baudelaire. Un poète lyrique à l'apogée du capitalisme, traduit de l'allemand par Jean Lacoste, Paris, Payot, «Petite bibliothèque Payot», 1982, pp. 55-98.

${ }^{7}$ „Die Sprache hat es unmißverständlich bedeutet, daß das Gedächtnis nicht ein Instrument für die Erkundung des Vergangenen ist, vielmehr das Medium“, Walter Benjamin, „Ausgraben und Erinnern“, Gesammelte Schriften, t. IV, 1, Frankfurt/Main, Suhrkamp, 1972, p. 400.
} 
dont les plus voyants sont les agences de voyages. Alors c'est de norme qu'il s'agit. Si dans le premier cas, on peut parler d'homotopies, dans le second, il s'agirait de normotopie, comme nous allons en développer les notions dans les chapitres suivants. En ce sens, la normalisation des sites mémoriels a pris la place où aurait pu se déployer une utopie que nous n'intégrons pas à la présente étude. Le troisième niveau s'avère certainement le plus riche: il s'agit des relations hétérogènes qui animent un espace regroupant des sites de nature différente. Là, la notion d'hétérotopie forgée et développée par Michel Foucault se révèle particulièrement éclairante.

\section{Homogénéisation et normalisation}

Peut-on encore ne pas être étonné par la ressemblance des architectures mémorielles? Les plus récentes semblent toutes avoir pris pour modèle la forme de l'anonymat des stèles funéraires. Ce constat prend sa source dans le rapprochement auquel engagent déjà certains mémoriaux avec des œuvres d'artistes dont l'impulsion critique et les créations, au tournant des années 1980-1990, ont constitué comme l'avant-garde informelle de la prise de conscience de la Shoah dans l'espace public (Shimon Attie, Christian Boltanski, Jochen Gerz, Horst Hoheisel, George Segal, Richard Serra, Micha Ullman, Rachel Whiteread...). Il est, par ailleurs, surprenant que la diversité des nationalités des architectes n'ait guère d'influence sur le canon qu'ils contribuent fortement à construire et à institutionnaliser. Pour la Belgique, le bâtiment du musée face à la Caserne Dossin a été conçu par le Flamand Bob Van Reethen ; à Varsovie, le musée Polin par les Finlandais Lahdelma \& Mahlamäki, à Drancy le musée mémorial par le Suisse Roger Diener ou celui du camp de Rivesaltes par le Français Rudy Ricciotti, la Topographie de la Terreur par l'architecte Ursula Wilms, du bureau d'architecture Heinle, Wischer und Partner de Berlin, le Mémorial de l'Holocauste par l'américain Peter Eisenman ou le musée Juif à Berlin par Daniel Libeskind, également américain... Tous ces bâtiments sont de formes cubiques, massives, sans fantaisie symbolisant, aujourd'hui, moins ce que James Young nommait des contre-monuments qu'un contre-lyrisme austère mais néanmoins monumental à l'image d'une mémoire historique qui, en vingt ans, s'est rapidement normalisée. La conformité esthétique de ces volumes définit une homotopie transnationale qui, tout en s'inscrivant d'emblée au nombre des nouvelles tendances générales de l'architecture, confère une autorité imposant le respect.

L'utilisation du Corten, cet acier auto-patiné dont on a forcé la corrosion, est également l'exemple flagrant d'une standardisation qui, illustrant la dégradation de la matière, participe d'une complète simulation référentielle. Car si rien mieux que le Corten ne peut représenter, à l'échelle de structures entières, l'idée de rouille et, partant, d'une altération par le temps signifiant le passé, alors qu'il s'agit, en réalité, d'un matériau d'une solidité extrême et, précisément, à l'épreuve du temps. La mémoire aurait-elle besoin d'un support qui résiste à l'usure pour être authentiquement représentée? La " rencontre » de l'austérité des grands cubes mémoriels et du brun grumeleux du Corten nous dit combien la référentialité par laquelle la mémoire se relie au passé, pour le reconfigurer, est une construction.

Mais l'homogénéité des formes ne suffit pas pour approcher les spécificités de ces nouveaux espaces occupés par la mémoire collective. Maîtriser ces rapports au passé pour asseoir les principes d'une mémoire normative constitue à l'évidence un enjeu pour les institutions, les entrepreneurs et les acteurs mémoriels. Ce que l'on pourrait désigner par le mot ingrat de normotopie repose évidemment sur des règles beaucoup plus complexes que la précédente homotopie, où s'articulent tendances globales et locales de nos monde(s) et de nos temps actuels, sans même qu'il soit toujours nécessaire qu'une "politique de la mémoire», 
intentionnelle ou concertée, soit clairement établie - le propre de la norme étant, pour la plupart des individus, de s'exercer naturellement et de se parer d'évidence. Si la recrudescence des sites mémoriels souligne que ceux-ci se situent au carrefour d'intérêts moraux, culturels et commerciaux à l'échelle mondiale, toutefois, chaque site peut être lui-même porteur d'enjeux politiques d'identité communautaire ou nationale très puissants qui constituent potentiellement une réaction à la normalisation globale de la mémoire, une réaction qui peut être identitaire et ultranationaliste comme le sont certains musées en Europe de l'Est ${ }^{8}$.

Le Musée de l'Insurrection de Varsovie (Muzeum Powstania Warszawskiego) de 1944, inauguré le 31 juillet 2004, est un des exemples les plus flagrants d'exaltation du sentiment national prenant position, dans l'espace symbolique de la ville, face au musée Polin, inauguré en 2013. La mise en avant de l'identité comme prisme normatif par lequel est délivrée une version nationale de l'histoire factuelle se note également dans des musées de l'Europe occidentale ou des États-Unis, mais de façon toutefois plus tempérée. Le Musée de l'Holocauste à Washington aborde l'histoire du génocide des Juifs par l'évocation de l'armée libératrice, le visiteur américain entre ainsi dans l'histoire du génocide par sa propre histoire. La coloration, le point de vue et, partant, la production d'une vision particulière de l'histoire comptent parmi les caractéristiques de nombreuses institutions mémorielles qui relèvent localement du politique et de l'idéologie, et non plus d'une esthétique ou d'une morale transnationale. Même s'il est certain que l'accent identitaire se soit nuancé sur les sites flamands de la mémoire belge, on peut néanmoins remarquer combien la guerre de 1914-1918, moment décisif pour la construction de la revendication autonomiste flamande, a été l'objet d'investissements considérables dans les musées de la Tour de l'Yser à Dixmude - traditionnellement lieu de rassemblement ultranationaliste - et d'In Flanders Field à Ypres, même si ce dernier met nettement en avant l'appartenance de la municipalité au réseau des villes pour la paix promu par l'UNESCO.

En revanche, à une centaine de kilomètres des précédents, du côté français, le Mémorial international de Notre-Dame-de-Lorette affiche délibérément par sa conception un parti pris œcuménique et rassembleur qui n'est pas moins ambigu que les exemples inverses. Inauguré le 11 novembre 2014 par François Hollande, il réunit par ordre alphabétique 579606 noms de morts au combat sur les 90 kilomètres de front du Nord-Pas-de-Calais entre 1914 et 1918, soit quarante nationalités. La réalité historique de ces hommes qui savaient très bien «ce pour quoi ils se sont battus, le fait qu'ils savaient très bien qu'ils étaient Allemands, Autrichiens, Australiens ou Gallois: tout cela va disparaître dans cette espèce d'anneau où l'on est tous frères dans la mort de masse» (BECKER, 2014). Cette volonté, en dépit de la réalité historique, est un exemple flagrant parmi les plus récents de l'imposition d'une norme mémorielle répondant à une politique publique.

\section{De l'hétérotopie}

Prenons maintenant des exemples à partir desquels la question de l'hétérotopie filtre entre homogénéisation et normalisation mémorielle. Quelques ouvres de Jochen Gerz participent de cette intuition topographique du tissu mémoriel. En 2000, avec une association humanitaire qui le met en rapport avec des sans-logis, il installe un dispositif nommé Les Mots de Paris. Il s'agit d'un abribus et d'une large plaque de verre servant de tronc pour recevoir des

\footnotetext{
${ }^{8}$ Voir à propos du musée de Gdańsk : Paweł Machcewicz, «Le Musée», in Mémoires en jeu, n 8, 2018-2019, HiverPrintemps, pp. 161-172; Delphine Bechtel et Luba Jurgenson (dir.), Le Tourisme mémoriel en Europe centrale, Paris, Petra, «Usages de la mémoire», 2009.
} 
dons situés entre la Cathédrale Notre-Dame et l'hôpital de l'hôtel Dieu, un des centres touristiques les plus actifs de la capitale reposant sur le cœur même de son histoire. Un an avant, son installation Künstlers Traum - Goethe in Buchenwald développe avec une grande acuité ce rapport hétérotopique touchant à la mémoire comme à l'histoire. Au cours d'une campagne d'affichage développée pendant six mois, l'artiste trace un portrait de la ville de Weimar. Car Weimar n'est pas seulement un centre important de la modernité allemande qui avait déjà été portée par l'esprit des Lumières, mais la ville est également un éminent lieu du passé nazi à cinq kilomètres duquel, sur les hauteurs de l'Ettersberg elles-mêmes auréolées par le romantisme, avait été implanté en 1937 le plus grand camp de concentration nazi: Buchenwald. Ces deux faces font de cet espace une sorte de Janus mémoriel dont les valeurs et contrevaleurs symboliques n'ont pas échappé à Gerz. D'un côté, le bijou de l'histoire des idées allemande et européenne (diesem Juwel deutscher und europäischer Geistesgeschichte), explique le guide de la page web de la ville (Goethe et Schiller, Herder et Wieland, Nietzsche, Gropius, Feininger, Klee...) ; de l'autre, la violence la plus brutale administrée rationnellement par le système SS. Gerz souhaitait, pour conclure son installation, relier la ville et le camp par un laser, consacrant ainsi le passage de la clarté à l'obscurité. À cette lecture mémorielle qui ne complaît pas aux images touristiques, s'ajoute Erfurt situé à moins de trente kilomètres des deux précédents pôles. La ville historique est elle-même doublement marquée pour la présence de Luther et, à sa périphérie, par le bâtiment transformé en musée depuis 2011 de l'entreprise Topf \& Sohn qui a construit, entre autres, les fours du complexe d'extermination d'Auschwitz-Birkenau.

L'originalité de l'œuvre in situ de Gerz permet précisément d'établir des distinctions. Lartiste tisse une relation critique, en évitant toute confusion, entre les deux faces de cet espace, parce que Weimar ne peut rester indemne de cette proximité qui n'est pas simplement géographique, ni même seulement thématique. En introduisant une dimension réflexive sur les passés de ces lieux, il incite à réfléchir sur cette histoire partagée qui est à chercher hors des lieux communs dont le cloisonnement est soigneusement entretenu. Ce faisant, il révèle un agencement hétérotopique jusque-là écarté qui incite à penser en termes éthiques : les œuvres de Gerz déconstruisent la suprématie esthétique exacerbée par les sociétés actuelles où la valeur de ce qui existe passe par la représentation. Or, précisément, les entrepreneurs touristiques et généralement mémoriels souhaitent que l'on identifie les lieux qu'ils promeuvent par et pour leur représentation et leur conformité à des normes, afin que chacun puisse facilement les reconnaître pour s'y repérer. Pour ces derniers, Weimar peut être liée à Buchenwald, car il ne serait certainement pas politiquement correct de ne pas aborder cette coprésence, mais Weimar doit rester une ville vitrine, parfaitement bien arrangée, et Buchenwald un lieu historique de la honte nationale, parfaitement localisé, engageant au recueillement. C'est en ce sens que la normalisation peut fonctionner avec la différence, mais surtout sans faire de celle-ci un facteur critique. Il n'est pas agent plus efficace de cette normotopie que les agences de voyages sachant à merveille mettre en lien des endroits fondamentalement improbables les uns pour les autres, sans pour autant déclencher une réflexion qui concerne le vivre ensemble et les valeurs qui le sous-tendent.

C'est ainsi que, suivant un principe du même ordre, se retrouvent sur les mêmes prospectus le camp muséifié d'Auschwitz I, les mines de sel de Wieliczka, et Cracovie, avec son château et Kazimierz, son quartier juif. La normotopie est soutenue par une argumentation qui défie le réel pour le transformer en indice d'attraction. Il n'est d'ailleurs pas étonnant que le camp de Fossoli en Italie, où les Juifs ont été regroupés à partir de fin 1943 pour être déportés à Auschwitz - équivalent donc de Drancy pour la France, de Dossin pour la Belgique, de Westerbork pour les Pays-Bas - ait été l'enjeu d'un étonnant projet d'installation d'un parc d'attractions (LEONI, 1990). Si, effectivement, les sites mémoriels, ni même la mémoire n'ont 
pas été inventés par les Tour operators, en revanche, le pragmatisme de ces derniers combiné au principe de la valorisation de l'identité patrimoniale parvient fort bien à investir la diversité des lieux pour les normaliser dans leur différence. Le travail de certains artistes sait alors introduire un écart critique qui permet, pour ainsi dire, de desceller l'hétérotopie des carcans homo- et normotopiques, cela ne signifie cependant pas que l'hétérotopie nécessite toujours de telles interventions pour être mise au jour.

La configuration urbaine de Lower Manhattan, pointe extrême de la presqu'île newyorkaise, s'est considérablement modifiée sous le choc des attentats du World Trade Center, puis sous l'impulsion du projet d'édifier à leur emplacement un mémorial, inauguré en septembre 2011, et un musée, ouvert en mai 2014. Même si les rives le long de l'Hudson étaient déjà très peuplées de mémoires, ce quartier dédié à la finance a été réinvesti d'une dimension et d'une valeur mémorielles ancrées dans l'actualité à l'occasion de l'attaque que les États-Unis ont subie sur leur territoire continental le 11 septembre 2001. On est à proximité de l'embarcadère pour la statue de la Liberté et pour Ellis Island, lieu historique de l'immigration, récemment mémorialisé ${ }^{9}$, mais avant de monter sur le ferry qui y conduit, on a tout loisir de longer les monuments à la Seconde Guerre mondiale, à la guerre de Corée de Mac Adams, le Mémorial des Vétérans du Viêt Nam et The Sphere du sculpteur allemand Fritz Koenig, initialement installée non loin des Twin Towers en 1971, puis déplacée en 2002 à Battery Park après avoir été endommagée lors de l'attentat. À quoi il faut ajouter que se tiennent, dans les environs, le Musée des Indiens d'Amérique, le Museum of Jewish Heritage architecturalement conçu comme un mémorial de l'Holocauste, le Mémorial des policiers new-yorkais morts en service et l'étonnant parc mémorial de la famine irlandaise situé au-dessus de North Cove à la hauteur de One World Trade Center (surnommé Freedom Center) au pied de laquelle s'étendent désormais les deux vastes bassins du mémorial de Ground Zero et le musée. Tout un périmètre de la complexité mémorielle invite à être arpenté alors qu'initialement l'espace était dédié à la haute finance. Ainsi, le Lower Manhattan est un exemple flagrant de la spécificité hétérotopique d'un espace qui combine, sans les homogénéiser, une variété d'entités mémorielles avec d'autres niveaux de réalité socio-professionnelle.

Foucault estime que ces emplacements «suspendent, neutralisent ou inversent l'ensemble des rapports qui se trouvent, par eux, désignés, reflétés ou réfléchis.» (FOUCAULT, 1984). Prendre en considération la valeur hétérotopique de ces espaces n'est pas sans conséquence comme on l'a signalé à propos des ouvres de Jochen Gerz. Un des caractères majeurs de la normalisation consiste à produire un discours narratif où prime toute une économie argumentative de la documentation (il faut expliquer) et de l'émotion (il faut ressentir) abordée plus haut. Or, l'hétérotopie suspend et neutralise ces fonctions discursives et narratives, elle présente d'autres agencements, d'autres logiques humaines aussi. Si l'hétérotopie sait «juxtaposer», dit Foucault, «en un seul lieu réel plusieurs espaces incompatibles», elle ne les unifie pas. Aucune utopie, aucune épopée ne peut naître de ces réseaux mémoriels involontaires et, certainement, aucune mémoire au sens de cette unification du passé qui s'est imposée aujourd'hui comme un discours dominant une partie de notre culture et de notre rapport à nous-mêmes. Une hétérotopie de cette sorte se dessine sur la ville quand la diversité des mémoires indifférentes les unes aux autres se trouve en voisinage sans pour autant qu'un lien cohérent les rattache. De nombreux quartiers, voire de nombreux territoires extra-urbains invitent à être considérés sous cet angle, et l'on s'apercevra vite que les regarder comme des hétérotopies vient suspendre - voire permet de déconstruire - la face et l'identité qui nous sont

\footnotetext{
${ }^{9}$ En effet, après avoir été laissé à l'abandon depuis les années 1950 et amplement vandalisé, le lieu a peu à peu été réinvesti à partir des années 1980 pour être ouvert, en 2001, en tant qu'American Family Immigration History Center, avec de nouveaux espaces et de notables changements thématiques et scénographiques en 2011.
} 
«naturellement» présentés. Berlin avec ses différentes mémoires nazie et communiste, criminelle et victimaire, d'occupation et d'émancipation alternative est un des centres mémoriels urbains les plus denses et dynamiques en Europe occidentale. De multiples pôles attirent des flux de populations touristiques comme, précisément, ce qui s'étend du Potsdamer Platz à la porte de Brandebourg en passant par le monument de l'Holocauste, et en se prolongeant, à l'opposé, vers le Musée juif ou la Topographie de la terreur reposant sur une partie des caves du siège de la Police secrète nazie, et bordée par des restes du Mur, à quoi s'ajoutent, de l'autre côté de la Leipziger Strasse, aussi bien les façades couvertes de grands panneaux rappelant les répressions des ouvriers le 17 juillet 1953, que le musée de la Stasi et celui de Check Point Charlie et ainsi de suite.

Certains quartiers de Barcelone se prêtent à un constat comparable, mais de ce côté-ci de l'Europe, il semble que cela se joue tout autant à l'échelle urbaine qu'à celle des territoires pyrénéens frontaliers entre les traces escarpées des passages par où les Espagnols ont fui et, du côté français, les camps d'internement dans lesquels ces derniers ont été détenus. On mesure l'ampleur du succès de ces territoires extra-urbains, donc leur lisibilité, au nombre d'éditions de guide touristique ou qui s'en donnent l'allure. Les hétérotopies mémorielles seraient ainsi des agencements involontaires que ne gouverne aucune visée politique ou idéologique, en revanche elles ont une portée éthique considérable. Elles entretiennent un certain sens multidirectionnel du passé, car s'il est certain que les mémoires du nazisme et du communisme à Berlin ne participent ni des mêmes traces ni des mêmes symboles, leur croisement confère au passé une qualité toute particulière.

\section{Ouverture}

Les musées et les mémoriaux cherchent, par des dispositifs optimisant les combinaisons entre scénographie et technique, à circonscrire entièrement l'objet auquel ils se consacrent. Ces institutions comptent ainsi parmi les opérateurs majeurs de ladite «mémoire collective» à la fabrication de laquelle elles participent pleinement. Elles la mettent en représentation sous des formes variées, attrayantes, ingénieuses et émouvantes qui prétendent tout en dire. En ce sens, que les frontières génériques entre musée, mémorial et monument soient de moins en moins perceptibles est un signe flagrant de cette évolution. Or, ces complexes mémoriels sont la plupart du temps observés, commentés et analysés comme des entités statiques et accomplies, ce qui restreint considérablement leur portée à l'échelle des espaces urbains et mentaux où ils prennent place. C'est pour aborder leur caractère évolutif et extensif au-delà de leurs murs que les différents concepts, heuristiques et provisoires, d'économie représentationnelle, d'homotopie, de normotopie et d'hétérotopie ont été introduits dans cette étude. Non pour leur faire nécessairement dire ce qui est, mais pour qu'ils ouvrent à une autre façon d'interroger la mémoire dans ses mouvements, sous ses masques et ses simulacres; une autre façon de percevoir avec acuité la façon dont la mémoire occupe, sous des formes très variées, les espaces que l'on habite.

\section{References}

BECKER, A. Tragédie vs catástrofe. Témoigner entre histoire et mémoire, Paris: Kimé, $\mathrm{n}^{\circ} 118$, septembre 2014, p. 44.

DE CERTEAU, M. L'Invention du quotidien, 1. Arts de faire. Paris: Gallimard, Folio essais, 1990, p. XXXVI, XLV sq. 
FOUCAULT, M. Histoire de la folie. Paris: Gallimard, 1972

FOUCAULT, M. Surveiller et punir. Naissance de la prison. Paris: Gallimard, 1975.

FOUCAULT, M. Citizens and Survivors: Cultural Memory and Oklahoma City. In: Des espaces autres. Dits et écrits II, Paris, Gallimard, 1984. LEONI, G. Trentecinque progetti per Fossoli. Milan: Electra, 1990.

MESNARD, P. La question du pathos dans les espaces des musées et des mémoriaux In: BECKER, A.; DEBARY, O. (dir.). Montrer les violences extremes. Paris: Creaphis, 2012.

STURKEN, M. Pilgrimages, Reenactment, and Souvenirs: Modes of Memory Tourism, In: HIRSCH, M.; MILLER, N. (dir.). Rites of Return. Diaspora Poetics and the Politics of Memory. New York: Columbia Press, 2011.

STURKEN, M. Tourist of History. Durham, N.C.: Duke University Press, 2007.

Recebido em: 08/07/2020

Aceito em: 27/07/2020 


\section{Documents complementaires}

Figura 1 - Sala central do Museu Historial da Grande Guerra. Péronne (França), 2016.

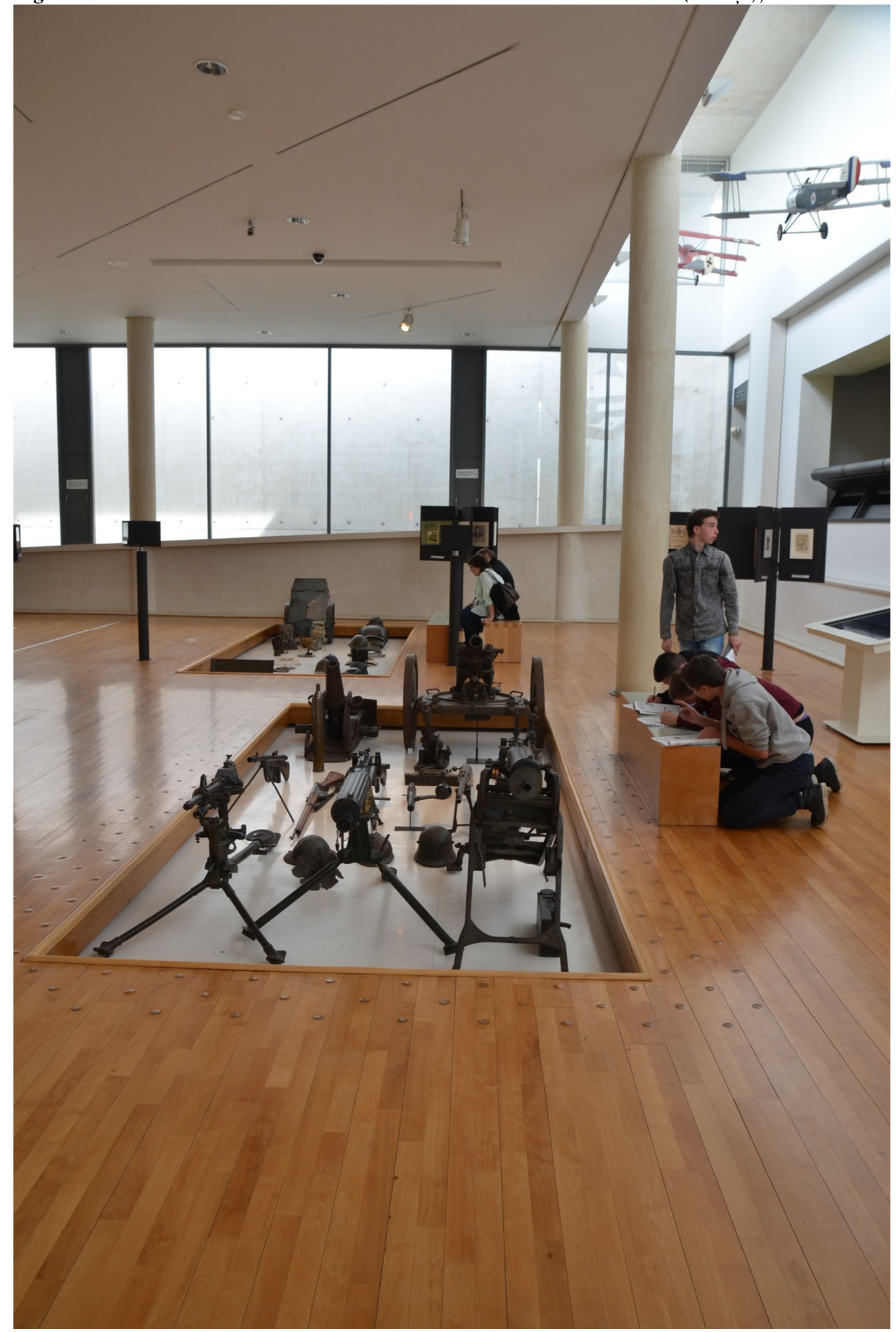

(C Philippe Mesnard) 
Figura 2 - MUME Museu de la Retirada (exílio dos Espanhóis ao final da Guerra Civil). La Junquera (Espanha), 2009.

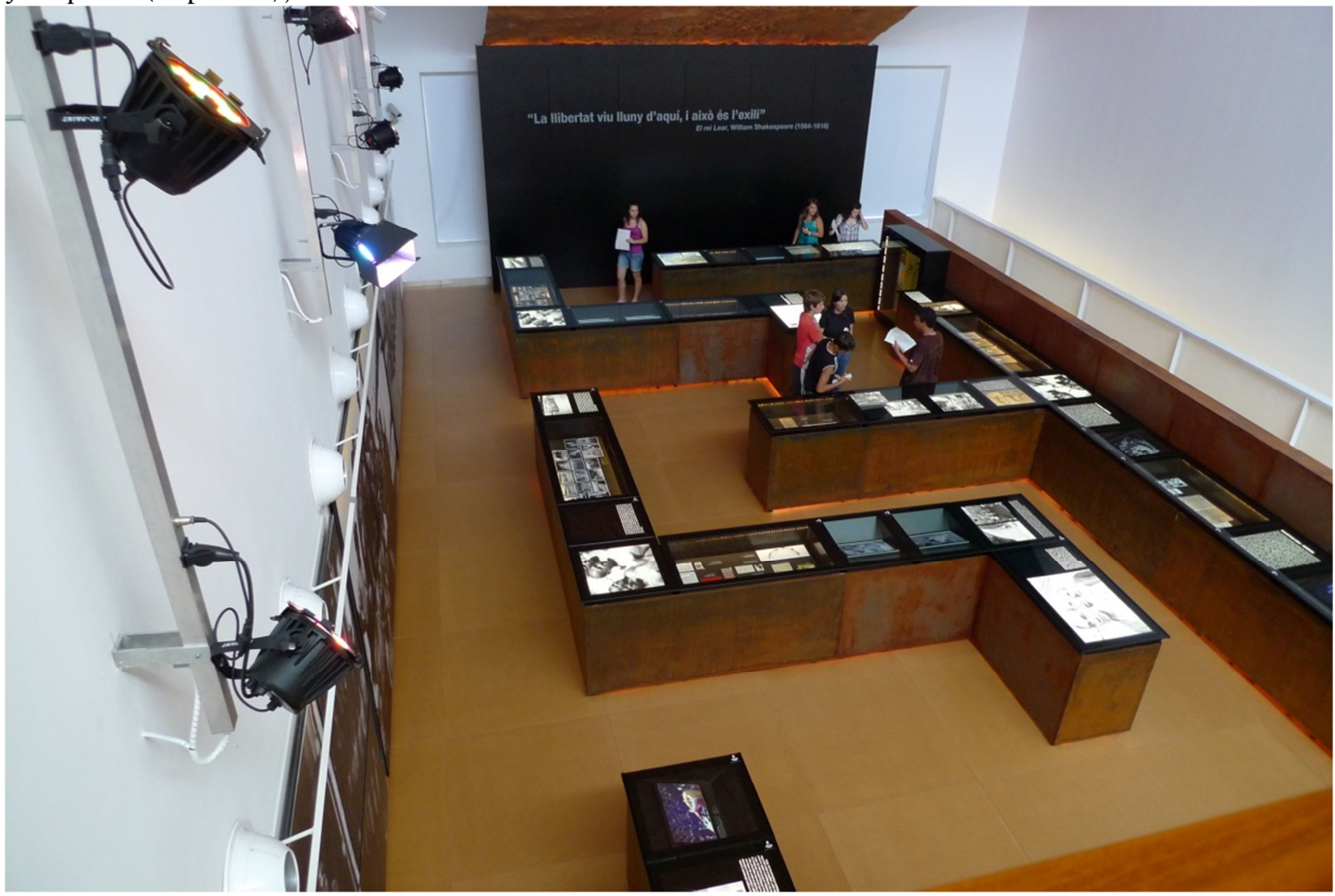

(C) Philippe Mesnard)

Figura 3 - Memorial das crianças de Yad Layeled, Kibbouts Lohamei Haghetaot (Israel), 2017.

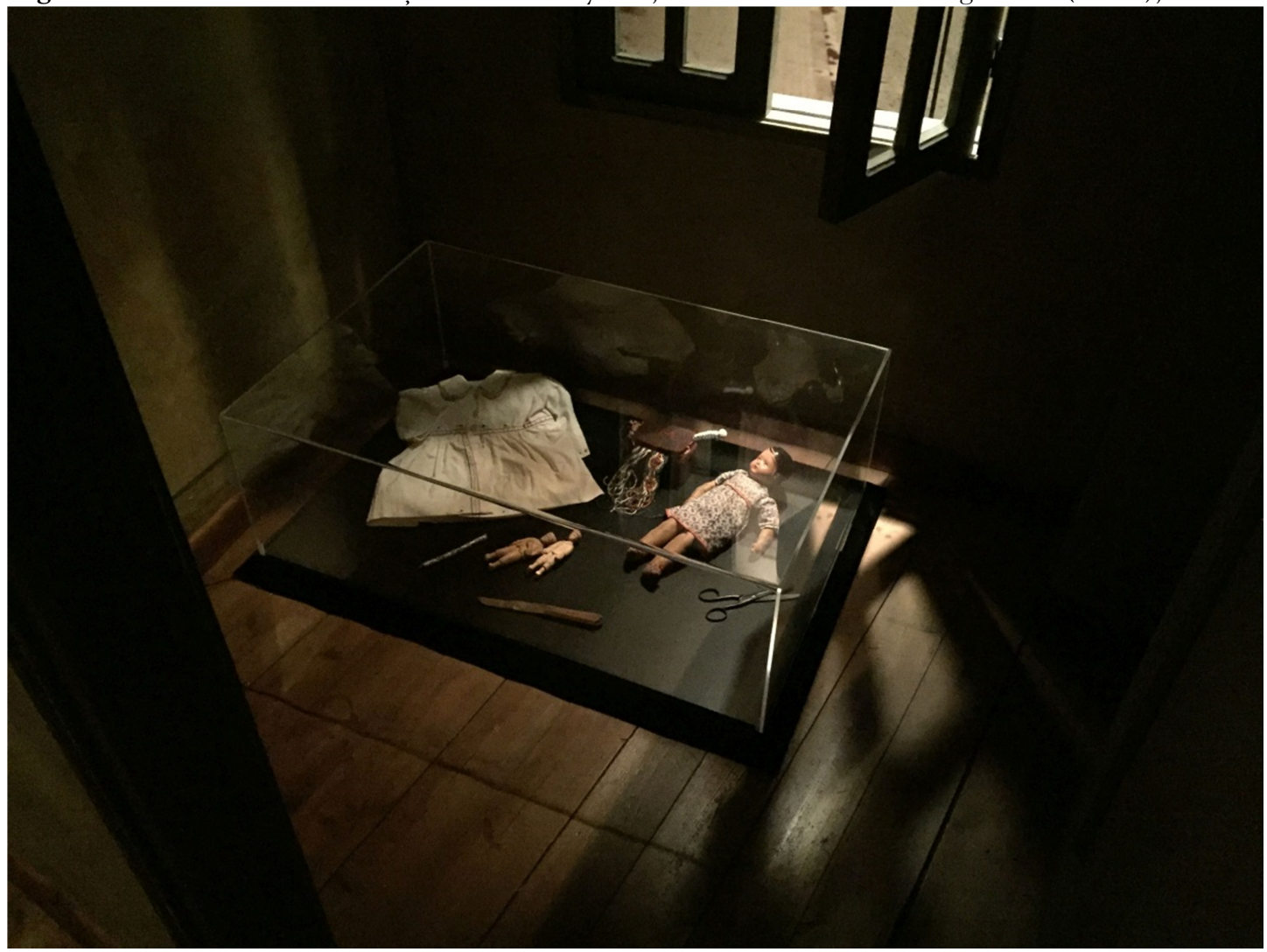

(C) Philippe Mesnard) 
Figura 4 - Museu de Katyń (nome de um dos lugares de massacre de tropas polonesas e principalmente de oficiais do exército polonês, pela NKVD, na primavera de 1940). 2019, Varsóvia, Polônia).

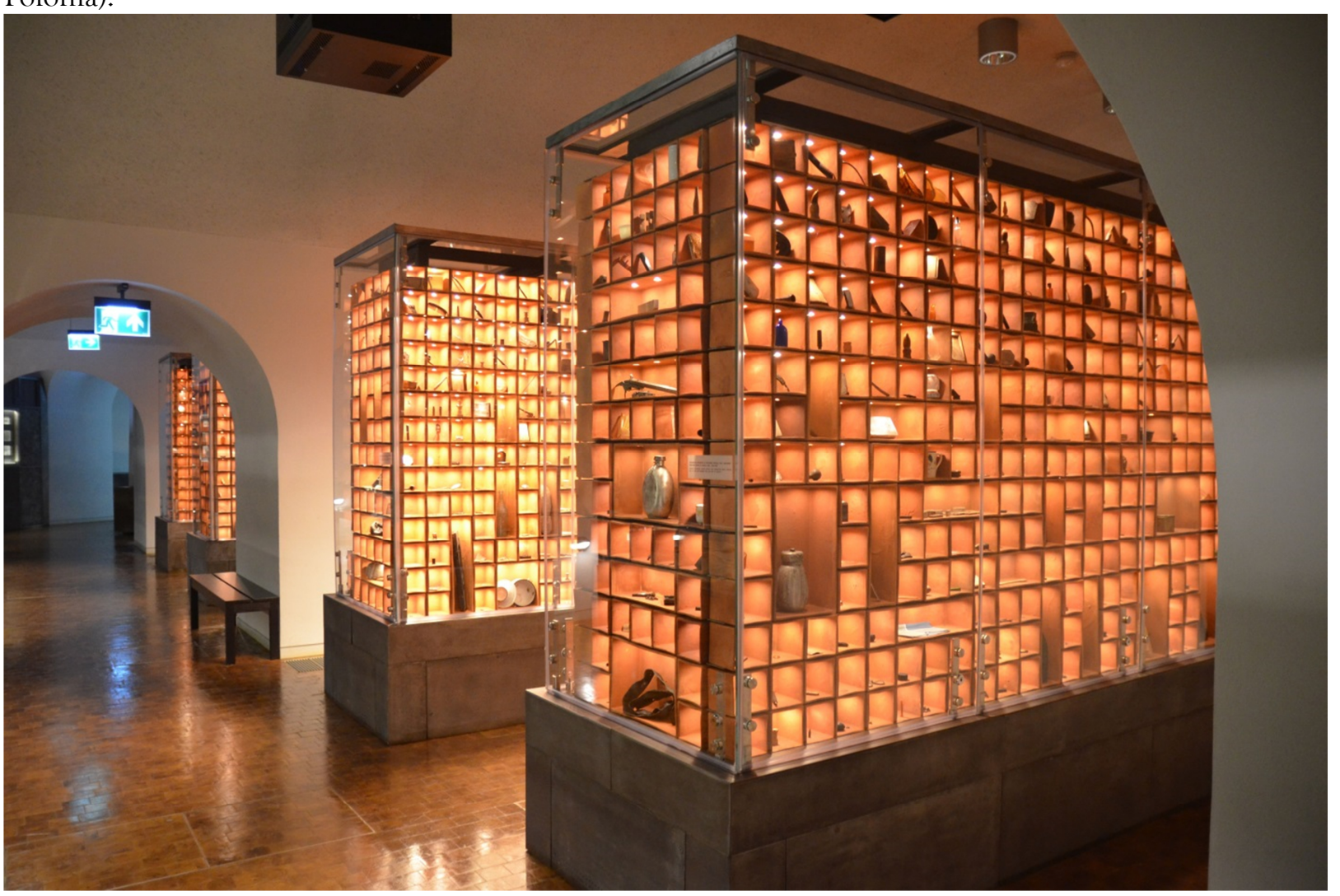

(C) Philippe Mesnard) 
Figura 5 - Ruínas conservadas no estado original. Memorial da cidade mártir Oradour-surGlane (França), 2018.

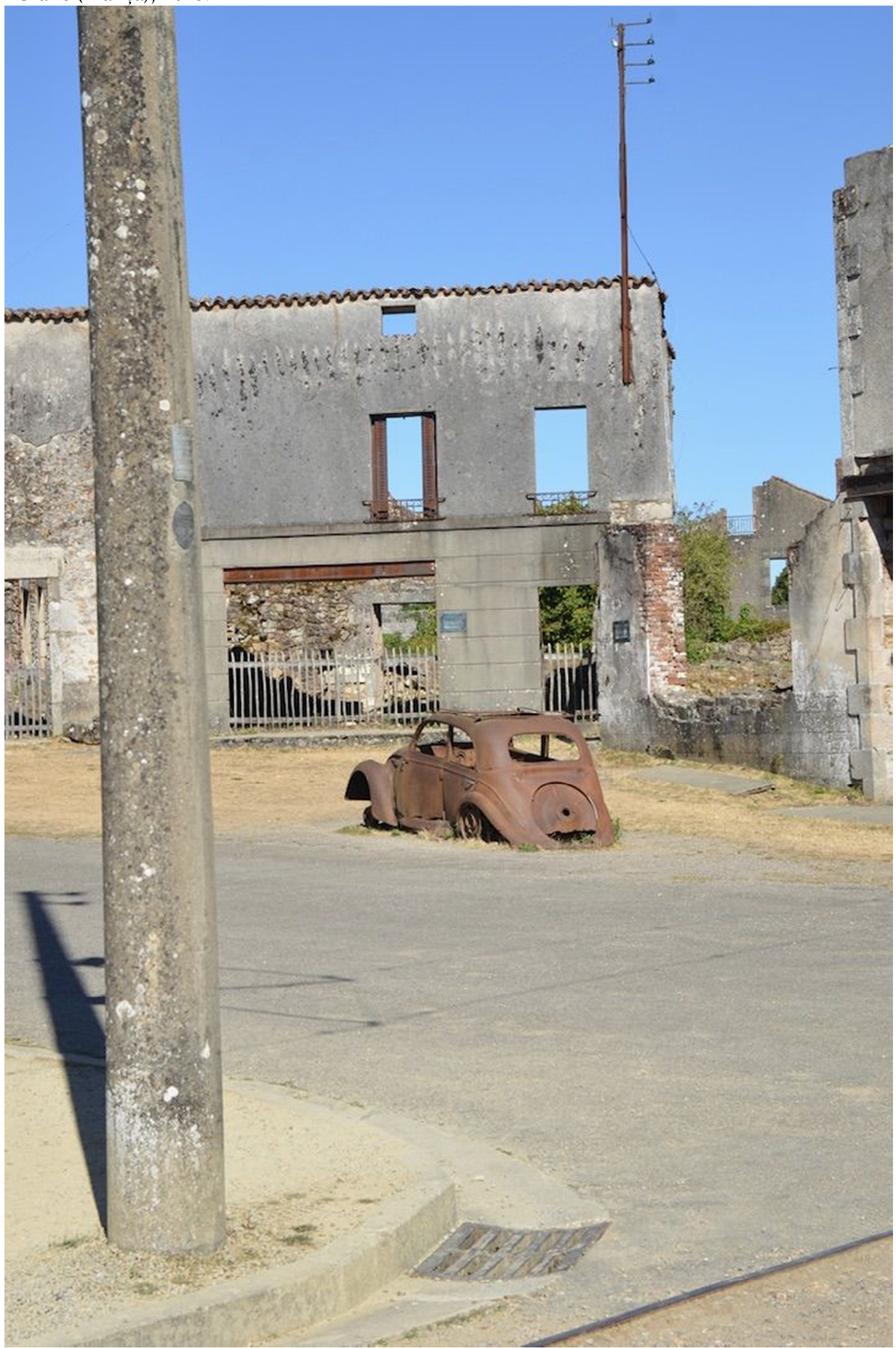

(C) Philippe Mesnard) 